\title{
SIMULATION OF TURBULENT WIND NOISE REDUCTION BY POROUS WINDSCREENS USING HIGH-ORDER SCHEMES
}

\author{
Y. XU \\ Key Laboratory of Microgravity, Institute of Mechanics \\ Chinese Academy of Science, Beijing, 100190, China \\ yingxu@imech.ac.cn \\ Z. C. ZHENG \\ Department of Aerospace Engineering \\ University of Kansas, Lawrence, Kansas, 66045 \\ zzheng@ku.edu \\ D. K. WILSON \\ US Army Cold Regions Research and Engineering Laboratory \\ Engineer Research and Development Center \\ Hanover, New Hampshire, 03755 \\ D.Keith.Wilson@usace.army.mil \\ Received 28 January 2010 \\ Revised 8 April 2010
}

\begin{abstract}
The purpose of the study is to investigate the wind noise reduction provided by microphone windscreens at different frequencies of the impinging turbulence. The windscreen is assumed to be a cylindrically shaped porous medium. This paper uses a high-order scheme to improve the accuracy at the interface between air and porous medium. The computational scheme is based on a modified immersed-boundary method with distributed forcing terms. The simulation results show that, for low-frequency turbulence, the windscreens with low flow resistivity are more effective in noise reduction, while for high-frequency turbulence, the windscreens with high flow resistivity are more effective.
\end{abstract}

Keywords: Wind noise; porous medium; high-order scheme; windscreen.

\section{Introduction}

This paper examines turbulent flow over a porous windscreen and the resulting attenuation of wind noise, i.e., the turbulent pressure fluctuations occurring on a microphone placed within the screen. Windscreens are widely used on microphones, particularly for outdoor acoustic measurements. In many such applications, wind noise interferes with the signals of interest, particularly at frequencies low in the audible range. The performance of measurement microphones thus heavily depends on correct windscreen designs. Production of 
wind noise inside a windscreen is a complicated aerodynamic noise problem because of the interaction with the atmospheric turbulence with the porous windscreen. Currently, windscreen design practice is mostly heuristic in nature. Therefore, there is a need to fully understand the mechanisms involved in flow/pressure fluctuations around a screened microphone. Such understanding can lead to optimized windscreen designs. In this study, the effects of turbulence and the windscreen material properties (specifically its flow resistivity) are investigated. Time-domain computational techniques are developed to study the detailed flow mechanisms around the windscreen as well as the flow inside the windscreen.

With the advent of time-domain methods, ${ }^{1}$ numerical simulations for acoustic problems can be combined with computational fluid dynamics. This provides powerful new tools to tackle acoustic problems. Recently, Wilson et al. used the finite-difference, time-domain (FDTD) method to simulate turbulence-induced pressure fluctuations around a porous microphone windscreen. ${ }^{2}$ The unsteady, incompressible fluid flow equations were solved outside the windscreen, whereas an incompressible form of the Zwikker-Kosten ${ }^{3}$ (ZK) equation was solved within the porous medium, which (as for the case of an impermeable, flexible membrane) resulted in a Laplace equation for the pressure field. However, the flow within the porous medium does not vanish. In this paper, an improved, coupled simulation is developed for solving these same equations.

Recently numerical simulations of flow over porous media have aroused much interest and attention. ${ }^{4}$ Specifically, the presence of a porous medium introduces a discontinuity at the interface between the fluid and porous medium, and also has been found to decrease computational accuracy. Therefore, accuracy at the interface is a key point in simulating such problems. Consequently, finding accurate and efficient ways to increase the accuracy and decrease the discontinuity at the interface is very important in simulations. One of the most effective ways to overcome the discontinuity is to apply high-order schemes such as upwind schemes or weighted essentially nonoscillatory (WENO) schemes. ${ }^{5,6}$

While stability of first-order upwind schemes is often acceptable, such schemes have a strong diffusive effect similar to the molecular viscosity. ${ }^{7}$ We thus consider and compare second-order upwind, third-order upwind, and fifth-order WENO schemes.

High-order schemes have previously been used for viscous flow around steady and moving solid bodies, ${ }^{8}$ but not for flow involving different types of media. Thus, in this paper, the high-order scheme will be used in the regions near the interface between fluid and porous media for the first time. The high-order scheme is combined with a modified immersedboundary method..$^{8,9}$

\section{Formulation and Numerical Schemes}

The two-dimensional model problem is shown in Fig. 1, where a stream of unsteady and/or turbulent flow approaches a cylindrical unscreened or screened microphone. The windscreen, when present, is made of a porous material. Because of the unsteadiness and surface conditions, flow fluctuations and vortical structures are generated around the surface and in the wake region. The pressure fluctuations sensed by the microphone, which is assumed to 


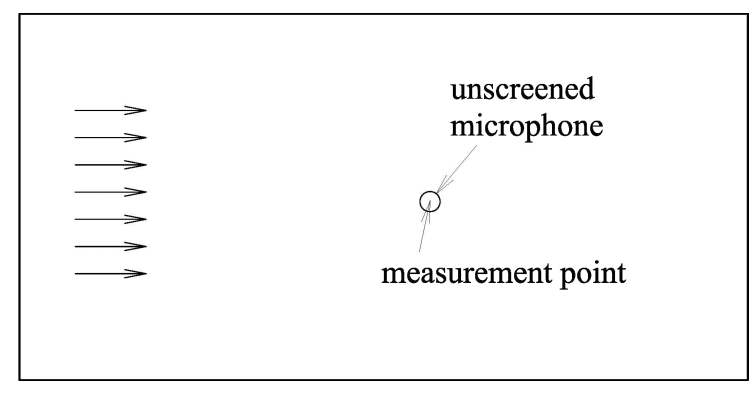

(a) Unscreened microphone

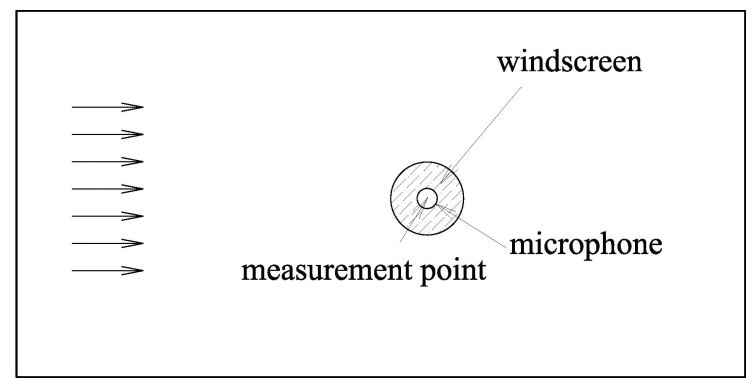

(b) Screened microphone

Fig. 1. Illustration of the model problem and the computational domain: (a) An unscreened microphone; (b) A screened microphone.

be at the center of the screen, result from near-field, incompressible disturbances. The flow fluctuations, both internal and external to the windscreen, are investigated based on coupled flow simulation between the outside and inside of the windscreen. We have developed an immersed-boundary (IB) computational method ${ }^{8,9}$ suitable for simulations in which a windscreen is immersed in a background flow. While the IB methods for fluid-structure interaction problems typically discretize the equations of motion for fluid on a Cartesian grid, the methods generally do not require that the geometry of the structure to conform in any way to this Cartesian grid.

The model equations are the Navier-Stokes (NS) equations for incompressible flow, with the modified ZK equation for flow inside the porous medium. It should be noted that although sound waves measured at a microphone are compressible disturbances, the wind noise interfering with the sound waves consist, in general, of incompressible turbulence. The pressure fluctuations of interest are near the surface of an object or inside a porous medium. These fluctuations are associated with near-field, as with the surface pressure fluctuations produced by a turbulent boundary layer over the surface (as discussed by Kraichnan ${ }^{10}$ ), for which the incompressible flow assumption is well justified.

For the convenience of numerical computation and flow characterization, the governing equations are nondimensionalized with the incoming wind speed, $\mathrm{U}$, the diameter of the cylindrical windscreen, $\mathrm{D}$, and the air density, $\rho$. The governing equations for the airflow for unsteady, incompressible flow can be generally written as:

$$
\frac{\partial u_{i}}{\partial t}+\frac{\partial}{\partial x_{j}}\left(u_{i} u_{j}\right)=-\frac{\partial p}{\partial x_{i}}+\frac{1}{\operatorname{Re}} \frac{\partial^{2} u_{i}}{\partial x_{j} \partial x_{j}}+f_{i}
$$

and

$$
\frac{\partial u_{j}}{\partial x_{j}}=0
$$

where all the variables are dimensionless, and the Reynolds number, Re, is defined as $U D / \nu$, with $\nu$ being the kinematic viscosity of the air. The body force, $f_{i}$, is a 
fictitious force that is introduced to enforce the flow outside the cylinder (either porous or solid) to accommodate the cylinder boundary condition, ${ }^{8}$ so that the flow both outside and inside the cylinder can be simulated using the same format of the governing equation.

We begin by discretizing the momentum equation Eq. (1) as:

$$
\frac{u_{i}^{n+1}-u_{i}^{n}}{\Delta t}=R H S_{i}+f_{i}
$$

where $R H S_{i}=-\left(\partial / \partial x_{j}\right)\left(u_{i} u_{j}\right)-\left(\partial p / \partial x_{i}\right)+(1 / \operatorname{Re})\left(\partial^{2} u_{i} / \partial x_{j} \partial x_{j}\right)$, and the $f_{i}$ is given by,

$$
f_{i}=\left\{\begin{array}{cl}
0 & \text { outside the windscreen } \\
\sigma u_{i} & \text { inside the windscreen } \\
-R H S_{I}+\left(v_{b i}^{n+1}-u_{i}^{n}\right) / \Delta t & \text { inside solid body }
\end{array}\right\}
$$

where $v_{b i}^{n+1}$ is the velocity of the solid body at the $n+1$ time step; therefore, the condition $u_{b i}^{n+1}=v_{b i}^{n+1}$ will be satisfied inside the solid body.

The airflow outside the windscreen is modeled with the incompressible NS equation expressed in Eq. (1) as $f_{i}=0$. For simplicity, the windscreen is assumed to have porosity and structure constant equal to 1 . The air flow inside the windscreen is modeled with the ZK equation, ${ }^{3}$ which is the low-frequency limit of more general forms of porous media equations. ${ }^{11}$ In addition, incompressibility is assumed for flow inside the porous medium. The governing equations for airflow inside the windscreen are expressed in Eq. (1) as $f_{i}=$ $\sigma u_{i}$, where $\sigma$ is the dimensionless flow resistivity of the porous medium, nondimensionalized by $\rho \mathrm{U} / \mathrm{D}$. The convection and diffusion terms are neglected in the original ZK equation because the velocity is low in the porous medium. We still retain them here so that the same solver can be used for both the NS equation and ZK equation, as the effect of convection and diffusion automatically becomes small when the velocity is low. Moreover, we also want to test the cases when the flow resistivity is small, and then the convection effect may not be very small. However, the Forchheimer term, which represents the flow resistivity caused by the second-order effect of the velocity, is neglected due to the small magnitude of velocity in the porous medium.

By applying a divergence operator to both sides of Eq. (1) and invoking the incompressibility condition of Eq. (2), a Poisson equation for the pressure can be obtained as

$$
\nabla^{2} p=-\frac{\partial}{\partial x_{i} \partial x_{j}}\left(u_{i} u_{j}\right)
$$

The presence of the porous media introduces a discontinuity in some of the flow variables or their derivatives around the flow/porous interface. Under these circumstances most conventional finite-difference schemes would generate spurious numerical oscillations. Here we introduce three approaches to addressing this problem: a second-order upwind scheme, a third-order upwind scheme, and a fifth-order WENO scheme. 
(i) Second-order upwind scheme $e^{12}$

Upwind schemes use an adaptive finite difference stencil to numerically simulate more properly the direction of propagation of information in a flow field. To outline this second order scheme, consider the simplified one-dimensional, one-way factored wave equation:

$$
\frac{\partial u}{\partial t}+a \frac{\partial u}{\partial x}=0
$$

Defining:

$$
\begin{gathered}
a^{+}=\max (a, 0) \quad a^{-}=\min (a, 0) \\
u_{x}^{-}=\frac{3 u_{i}-4 u_{i-1}+u_{i-2}}{2 \Delta x} \\
u_{x}^{+}=\frac{-u_{i+2}+4 u_{i+1}-3 u_{i}}{2 \Delta x}
\end{gathered}
$$

We can discretize the second term of Eq. (6) as:

$$
\left.a \frac{\partial u}{\partial x}\right|_{x=x_{i}^{n}}=a^{+} u_{x}^{-}+a^{-} u_{x}^{+}
$$

(ii) Third-order upwind scheme $e^{12}$ :

Defining:

$$
\begin{aligned}
& u_{x}^{-}=\frac{2 u_{i+1}+3 u_{i}-6 u_{i-1}+u_{i-2}}{6 \Delta x} \\
& u_{x}^{+}=\frac{-u_{i+2}+6 u_{i+1}-3 u_{i}-2 u_{i-1}}{6 \Delta x}
\end{aligned}
$$

Substituting Eqs. (10) and (11) into Eq. (9), we can get the expression for the third-order upwind scheme.

(iii) Fifth-order WENO scheme

To outline this fifth-order scheme, consider the one-dimensional wave equation in the form of

$$
\frac{\partial u}{\partial t}+\frac{\partial q(u)}{\partial x}=0
$$

The derivative of any flux, $q$, is discretized as:

$$
\left.\frac{\partial q}{\partial x}\right|_{x=x_{i}}=q_{x, i}=\frac{\hat{q}_{i+1 / 2}-\hat{q}_{i-1 / 2}}{\Delta x}
$$

where $\hat{q}_{i+1 / 2}$ is an interpolated flux at the half-step location.

If $\partial q / \partial u \geq 0$

$$
\begin{aligned}
\hat{q}_{i+1 / 2}^{+}= & \omega_{1}\left(\frac{1}{3} q_{i-2}-\frac{7}{6} q_{i-1}+\frac{11}{6} q_{i}\right)+\omega_{2}\left(-\frac{1}{6} q_{i-1}+\frac{5}{6} q_{i}+\frac{1}{3} q_{i+1}\right) \\
& +\omega_{3}\left(\frac{1}{3} q_{i}+\frac{5}{6} q_{i+1}-\frac{1}{6} q_{i+2}\right)
\end{aligned}
$$


where

$$
\begin{aligned}
& \omega_{j}=\frac{\alpha_{j}}{\sum_{k=1}^{3} \alpha_{k}}, \quad \alpha_{k}=\frac{d_{k}}{\left(\varepsilon+\beta_{k}\right)^{2}} \\
& d_{1}=\frac{1}{10}, \quad d_{2}=\frac{3}{5}, \quad d_{3}=\frac{3}{10}, \quad \varepsilon=10^{-6} \\
& \beta_{1}=\frac{13}{12}\left(q_{i-2}-2 q_{i-1}+q_{i}\right)^{2}+\frac{1}{4}\left(q_{i-2}-4 q_{i-1}+3 q_{i}\right)^{2} \\
& \beta_{2}=\frac{13}{12}\left(q_{i-1}-2 q_{i}+q_{i+1}\right)^{2}+\frac{1}{4}\left(q_{i-1}-4 q_{i+1}\right)^{2} \\
& \beta_{3}=\frac{13}{12}\left(q_{i}-2 q_{i+1}+q_{i+2}\right)^{2}+\frac{1}{4}\left(3 q_{i}-4 q_{i+1}+q_{i+2}\right)^{2}
\end{aligned}
$$

More details about the WENO scheme can be found elsewhere. ${ }^{5,6,8}$

For all of the considered schemes, Eq. (1) is discretized using the first-order time marching, with a semi-implicit scheme for the diffusion terms and the second-order AdamsBashforth scheme for convection and central differencing for diffusion. The high-order schemes discussed above are only used for the convection term in the region around the flow/porous interface. In the present study, the region is two times as big as the porous area. Continuity is enforced by solving the Poisson equations for pressure, Eq. (5), using a fast Poisson solver. ${ }^{13}$ A more detailed explanation of the IB computational scheme can be found in Ref. 9. It should be noted that since we intend to capture all of the flow fluctuations, no turbulence models are used in the simulation.

\section{Validation Study}

Low Reynolds number flow over a stationary solid cylinder is used as a validation study case to test the three flux derivative schemes. The numerical model for the cylinder case is twodimensional and time-dependent, and nondimensionalized by the diameter of the cylinder $\mathrm{D}$ and the free-stream velocity $\mathrm{U}$. The computation is performed on a staggered Cartesian grid mesh.

For the boundary conditions, at the inlet, $u=1, v=0$, and $\partial p / \partial x=0$. For the bottom and top, $\partial u / \partial y=0, v=0$, and $\partial p / \partial y=0$. For the outlet, $\partial u / \partial x=0, \partial v / \partial x=0$ and $p=0$. The domain size is $25.6 \times 12.8$, with the grid size set to $d x=d y=0.025$. The Reynolds number is 40 and the time step is 0.004 . The center of the cylinder is located 8 unit lengths from the inlet.

The validation is done by investigating the surface-pressure distribution on the cylinder surface, which is most sensitive to different flux derivative schemes. For Reynolds number less than 50, a pair of attached, steady and symmetric recirculation zones exist downstream of the cylinder. Since no vortex shedding has been formed, the pressure coefficient $\mathrm{Cp}$ on the surface is steady. Figure 2 shows the $\mathrm{Cp}$ on the cylinder surface at $\mathrm{Re}=40$. For reference, the three implemented schemes are compared with results from other studies. ${ }^{7,14,15}$ All are 


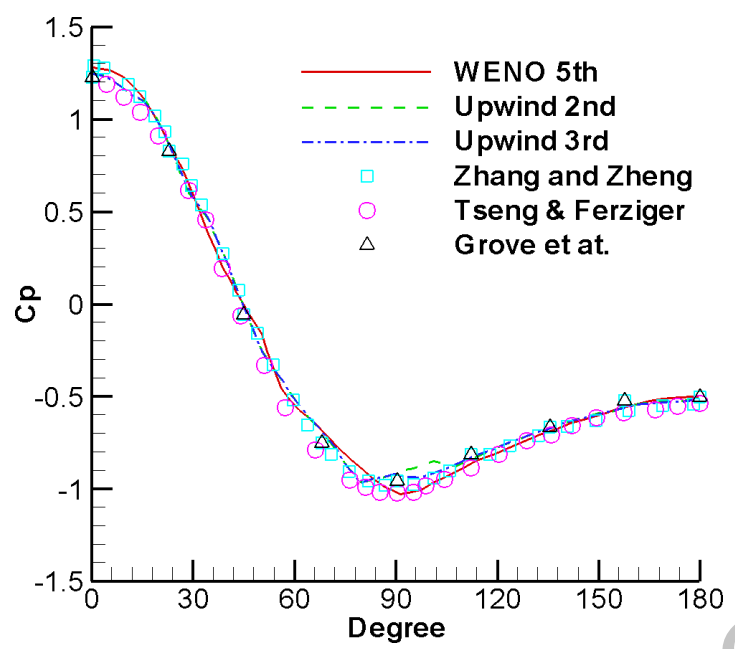

Fig. 2. Cp on the solid stationary cylinder surface, $\mathrm{Re}=40$, with different computational schemes.

in good agreement. We can see that the simulation with the fifth-order WENO scheme matches the reference results best. The third-order and second-order schemes have very similar shape, except for a few points near $100^{\circ}$. From this validation case, we can see that the high-order schemes are well implemented. In the following results we use the third-order upwind scheme as our basic simulation method, because it is easily coded and cost effective for computing time.

\section{Results and Discussions}

As mentioned previously, the simulation performed here applies time-domain computational fluid dynamics methodologies to an acoustic problem.

To distinguish the noise reduction effect of the windscreen in different frequency ranges, we introduce wind turbulence by placing different sizes of solid cylinders upstream of the microphone (Fig. 3). We selected cylinder of sizes $0.2 \mathrm{D}, 0.5 \mathrm{D}, 1.0 \mathrm{D}$ and $2.0 \mathrm{D}$ to generate different turbulent fields (where $\mathrm{D}$ is the diameter of the windscreen). The distance $\mathrm{L}$,

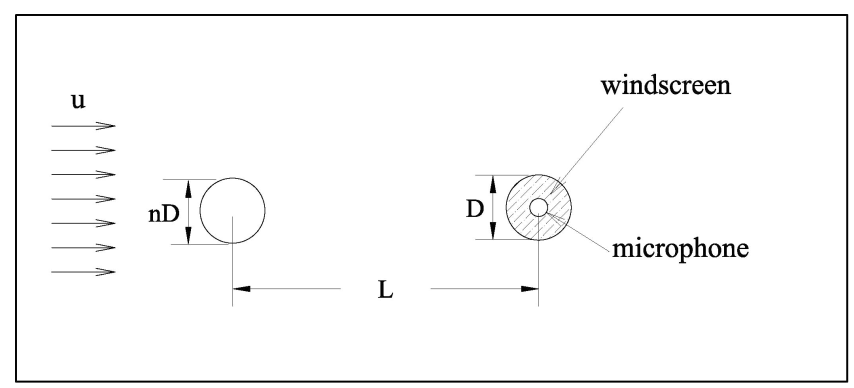

Fig. 3. Illustration of the incoming wind turbulence generation with different sizes of upstream cylinders where $n=0.2,0.5,1.0$, and 2.0 . 
between the first cylinder center and the second windscreen center, is 8D. More realistic atmospheric turbulence could be incorporated in future studies using other methods such as the quasi-wavelet method. ${ }^{16,17}$

The Reynolds number for the cases presented here is 500, which approximately corresponds to a windscreen with a diameter of $0.75 \mathrm{~cm}$, a bare microphone with a diameter of $0.1875 \mathrm{~cm}$, and a wind speed of $1 \mathrm{~m} / \mathrm{s}$ for the incoming air. The four chosen values of dimensionless flow resistivity of the windscreen, 0.2, 2, 20 and 200, correspond to dimensional flow resistivity values of approximately $34,340,3400$ and $34000 \mathrm{~Pa} \cdot \mathrm{s} / \mathrm{m}^{2}$, respectively $\left(1 \sigma=\rho U / D=1.27 \mathrm{~kg} / \mathrm{m}^{3} \cdot 1 \mathrm{~m} / \mathrm{s} / 0.0075 \mathrm{~m}=170 \mathrm{~Pa} \cdot \mathrm{s} / \mathrm{m}^{2}\right)$. In the simulation here, the grid size is 0.025 in both the $x$ and $y$ directions, and the time step is 0.002 , which satisfies the stability condition for the computational scheme. ${ }^{9}$

We first investigate the whole flow field as shown by vorticity contours in Fig. 4 for the flow resistivity $\sigma=340 \mathrm{~Pa} \cdot \mathrm{s} / \mathrm{m}^{2}$ with different upstream cylinder sizes $(0.2 \mathrm{D}, 0.5 \mathrm{D}, 1.0 \mathrm{D}$ and $2.0 \mathrm{D}$ case). The red color in Fig. 4 represents positive vorticity (in the counter clockwise sense, with the upper limit value of 5 ), the blue color represents negative vorticity (in the clockwise sense, with the lower limit value of -5). Clearly, smaller upstream cylinders generate smaller but denser vortical structures compared with larger upstream cylinders. Also, there is no flow inside the solid bare microphone but some flow infiltrates the windscreen porous cylinder. The vortical structures shed from the upstream cylinder are diffused by

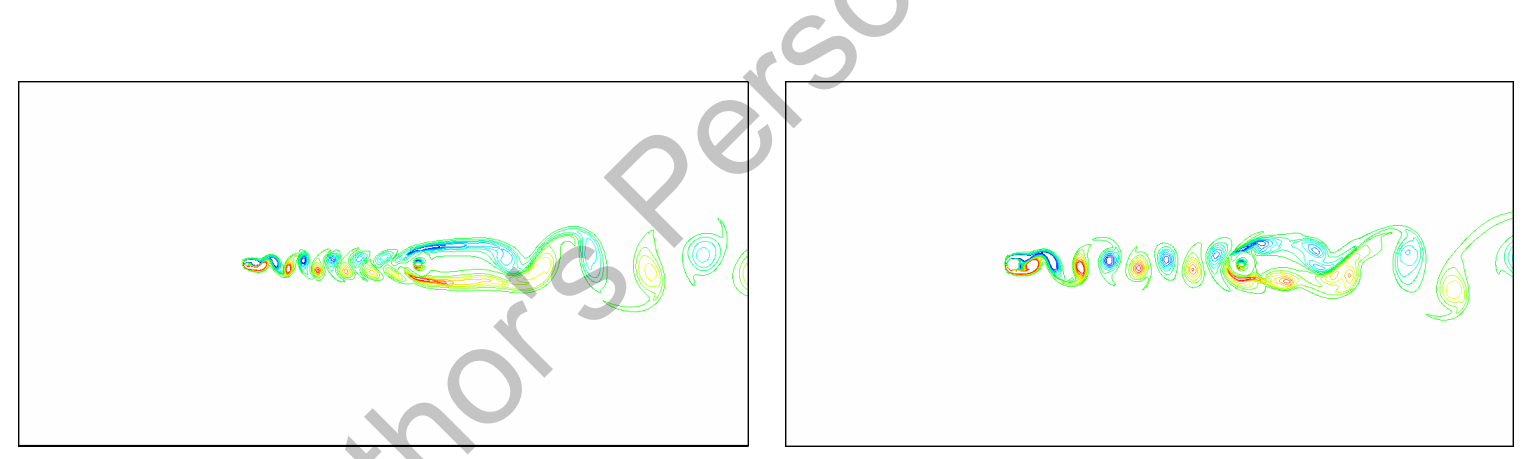

(a) $0.2 \mathrm{D}$

(b) $0.5 \mathrm{D}$
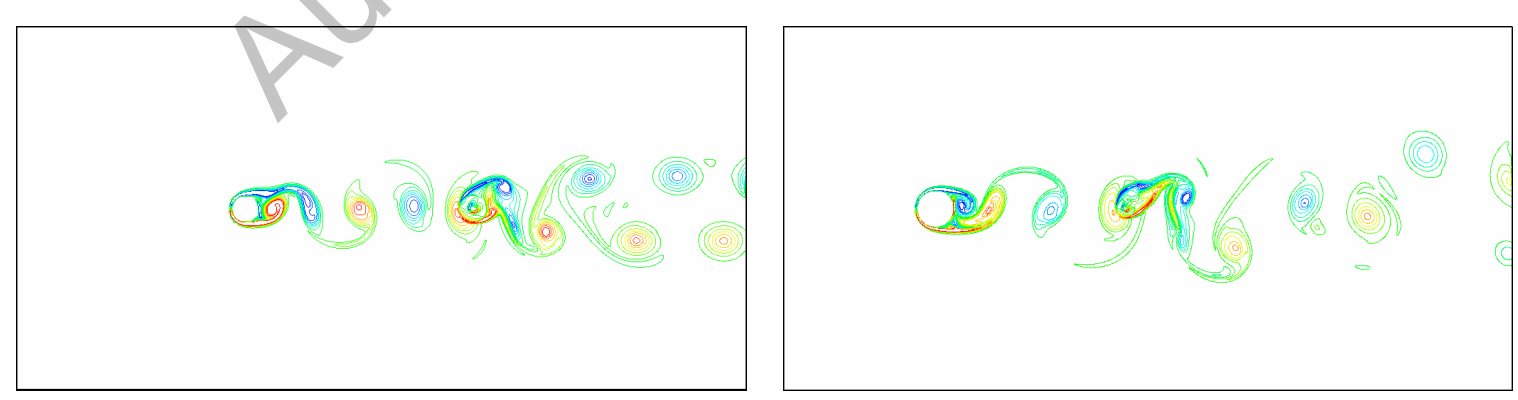

(c) $1.0 \mathrm{D}$

(d) $2.0 \mathrm{D}$

Fig. 4. (Color online) Vorticity contours of the flow field, with 1D-size downstream porous windscreen having flow resistivity $\sigma=340 \mathrm{~Pa} \cdot \mathrm{s} / \mathrm{m}^{2}$ and varying sizes of the upstream cylinder: (a) 0.2D, (b) 0.5D, (c) 1.0D, and (d) 2.0D. 


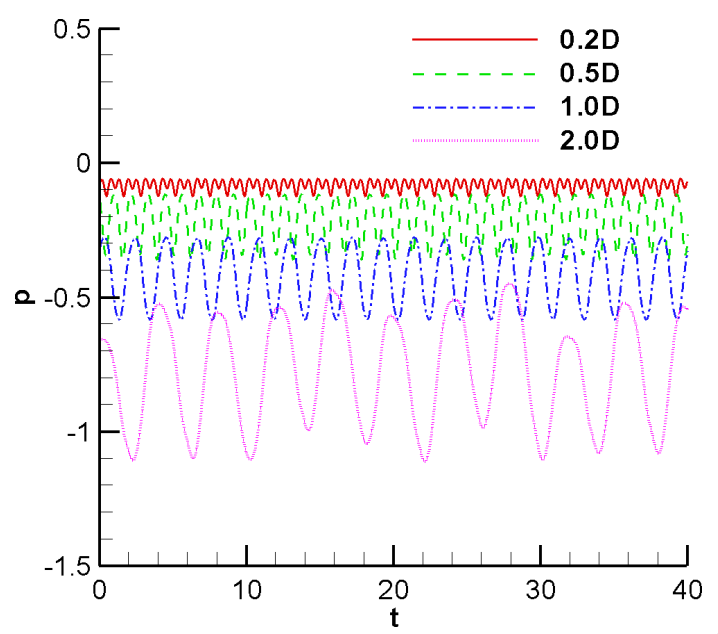

Fig. 5. (Color online) Pressure histories at a point downstream from the first solid cylinder. The measurement point is $4 \mathrm{D}$ away from first cylinder center, and all values are dimensionless.

the porous medium windscreen. Next, when the flow resistivity reaches high enough values $\left(\sigma=34000 \mathrm{~Pa} \cdot \mathrm{s} / \mathrm{m}^{2}\right)$ little flow permeates the porous cylinder, similar to flow over an unscreened bare cylinder. Finally, with very low flow resistivity (less than $10 \mathrm{~Pa} \cdot \mathrm{s} / \mathrm{m}^{2}$ ), the flow permeates the porous cylinder as if the porous medium layer does not exist.

Figure 5 shows pressure time histories at a point downstream from the first solid cylinder. The measurement point is 4D away from the center of the upstream cylinder center. The four curves are for four different upstream cylinder sizes. The time histories indicate that each case reaches a quasi-periodic state. Apparently, the smallest cylinder (0.2D) generates the highest frequency fluctuations and the largest cylinder (2.0D) generates the lowest frequency fluctuations. Meanwhile, the power spectrum density (PSD) comparison in Fig. 6 clearly

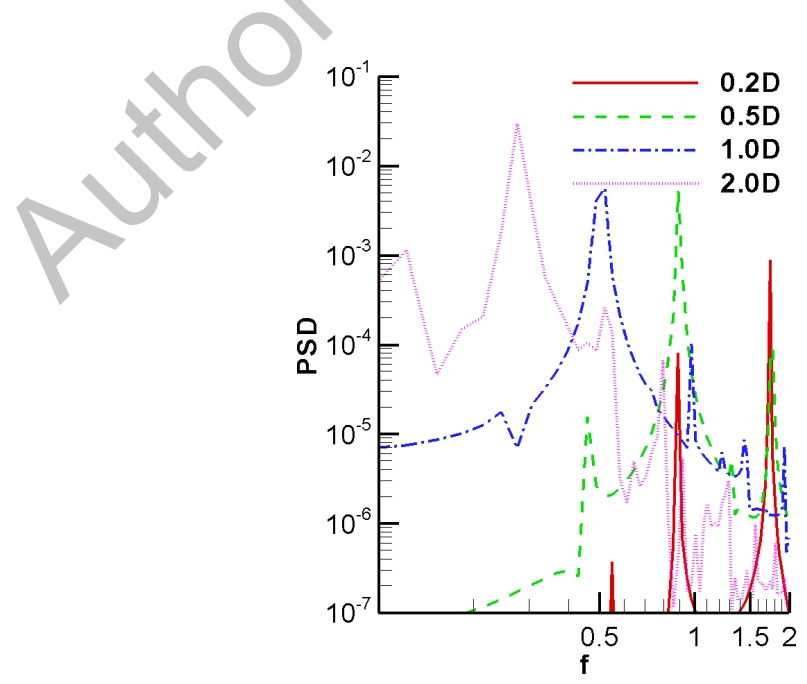

Fig. 6. Power spectral density (PSD) at a point downstream of the first solid cylinder. The measurement point is $4 \mathrm{D}$ away from the first solid cylinder center, all values are dimensionless. 
shows that the larger upstream cylinder generates lower-frequency perturbations in the flow field. Also notable is that a larger upstream cylinder generates a stronger signal because this cylinder has larger vortical structures (as shown in Fig. 4). The peak dimensionless frequencies, for the cases of $0.2 \mathrm{D}, 0.5 \mathrm{D}, 1.0 \mathrm{D}$, and $2.0 \mathrm{D}$, are respectively $1.8,0.9,0.5$, and 0.25 , resulting in a Strouhal number $(f D / U)$ ranging from 0.36 to 0.5 . We also found that the same size upstream cylinder generates almost the same downstream vortical structures regardless of the flow resistivity of the downstream windscreen cylinder. Therefore, with sufficient separation distance in this study, the upstream turbulence is not altered by the downstream condition.

Figure 7 shows the sound pressure level (SPL) at the center of the windscreen. The SPL is defined as $S P L=10 \log _{10}\left(f_{r} S_{p}(f) / p_{r}^{2}\right.$, where $f_{r}$ equals $1 \mathrm{~Hz}, P_{r}$ equals $20 \mu \mathrm{Pa}, S_{p}(f)$ is the PSD. Four different upstream cylinder cases are presented $(0.2 \mathrm{D}, 0.5 \mathrm{D}, 1.0 \mathrm{D}$ and 2.0D), and for each case five curves are shown: one for the bare microphone and the other four for the screened microphone with four different flow resistivities: 34, 340, 3400, and $34000 \mathrm{~Pa} \cdot \mathrm{s} / \mathrm{m}^{2}$. For the upstream cylinder of diameter 0.2D, Case (a), the SPL with high flow resistivity $\left(340,3400,34000 \mathrm{~Pa} \cdot \mathrm{s} / \mathrm{m}^{2}\right)$ is greater than that with the bare microphone case in the frequency range below $80 \mathrm{~Hz}$, which means that the windscreen has no reduction effect in this range. However, the windscreen is effective in the frequency range of $100 \mathrm{~Hz}$ to $400 \mathrm{~Hz}$ for Case (a). For Case (b) and Case (c) with the upstream cylinder diameters $0.5 \mathrm{D}$ and $1.0 \mathrm{D}$ respectively, it shows these two cases have similar windscreen effects, with windscreens effective in the range from $60 \mathrm{~Hz}$ to $400 \mathrm{~Hz}$. For Case (d) with the upstream cylinder diameter 2.0D, the SPL shape is similar among all five curves. Figure 7 also shows that the SPL shape of the lowest windscreen flow resistivity case $\left(\sigma=34 \mathrm{~Pa} \cdot \mathrm{s} / \mathrm{m}^{2}\right)$ is very similar to that of the unscreened microphone case except for very low frequency (below roughly $30 \mathrm{~Hz}$ ), which means that the low flow resistivity screen case is not discernibly different from the bare microphone case. It should be noted that the results at roughly $300 \mathrm{~Hz}$ and higher are likely artifacts of the windowing used in the FFT process.

We further investigate wind noise reduction level (WNR), defined as: $W N R=$ $S P L_{\text {unscreened }}-S P L_{\text {screened }}$, by subtracting the SPL at screened microphone center from that at unscreened microphone center. Figure 8 shows the WNR for four different upstream cylinder cases $(0.2 \mathrm{D}, 0.5 \mathrm{D}, 1.0 \mathrm{D}$ and $2.0 \mathrm{D})$, each with four curves showing results for the bare microphone minus results for each of the four screened microphones with varying flow resistivity $\left(34,340,3400,34000 \mathrm{~Pa} \cdot \mathrm{s} / \mathrm{m}^{2}\right)$. It can be seen that the highest WNR is around $40 \mathrm{~dB}$, which is a very significant reduction. Clearly, the WNR between the unscreened microphone and the lowest flow resistivity $\left(\sigma=34 \mathrm{~Pa} \cdot \mathrm{s} / \mathrm{m}^{2}\right.$, red dash line) case is smaller than the other three higher flow resistivity screens $\left(\sigma=340,3400,34000 \mathrm{~Pa} \cdot \mathrm{s} / \mathrm{m}^{2}\right)$. For Case (a), with the upstream cylinder diameter $0.2 \mathrm{D}$, the lowest flow resistivity windscreen $\left(\sigma=34 \mathrm{~Pa} \cdot \mathrm{s} / \mathrm{m}^{2}\right)$ performs better around a few tens of $\mathrm{H}$ at $30-100 \mathrm{~Hz}$, while the second lowest flow resistivity windscreen $\left(\sigma=340 \mathrm{~Pa} \cdot \mathrm{s} / \mathrm{m}^{2}\right)$ performs better around a few hundred Hz. For Case (b) with upstream cylinder diameter 0.5D, the medium flow resistivity windscreen $\left(\sigma=3400 \mathrm{~Pa} \cdot \mathrm{s} / \mathrm{m}^{2}\right)$ performs better around $70 \mathrm{~Hz}$ and the highest flow resistivity windscreen $\left(\sigma=34000 \mathrm{~Pa} \cdot \mathrm{s} / \mathrm{m}^{2}\right)$ performs better around a few hundred $\mathrm{Hz}$. 


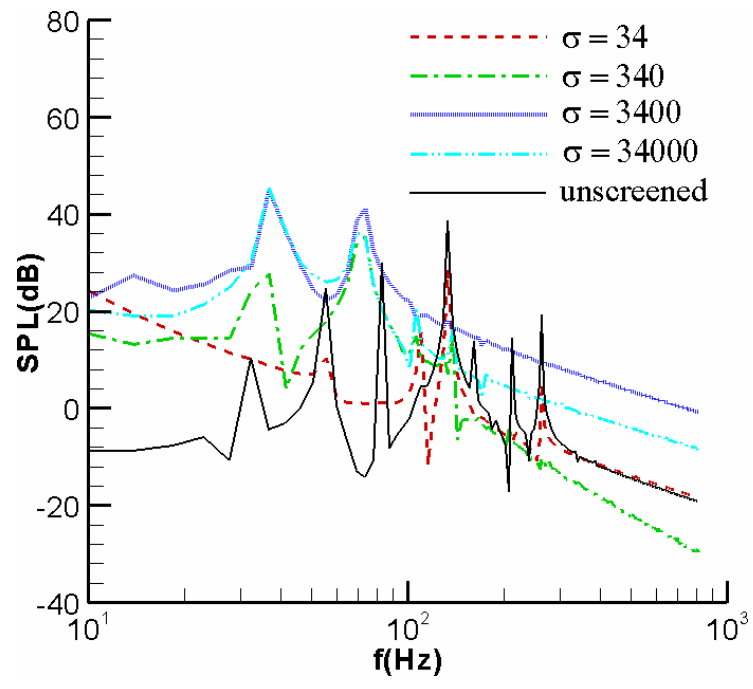

(a) $0.2 \mathrm{D}$

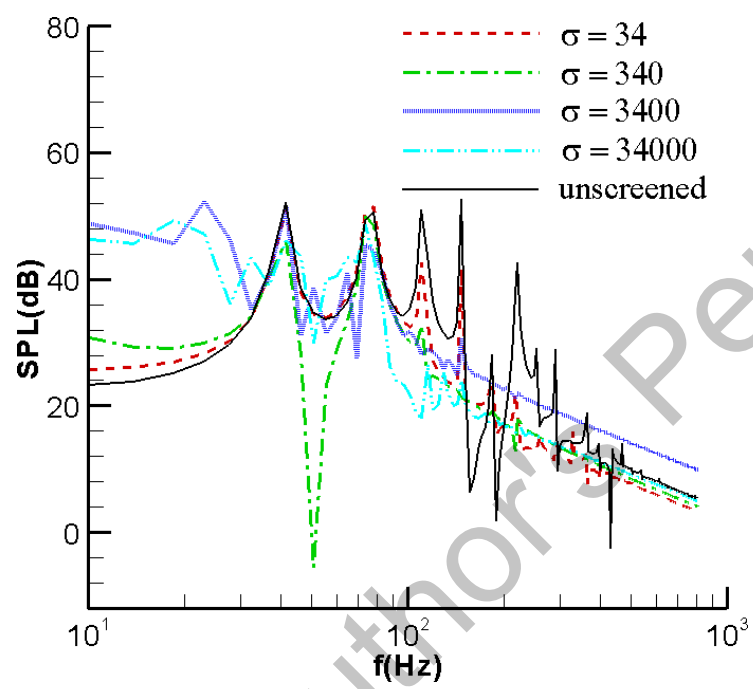

(c) $1.0 \mathrm{D}$

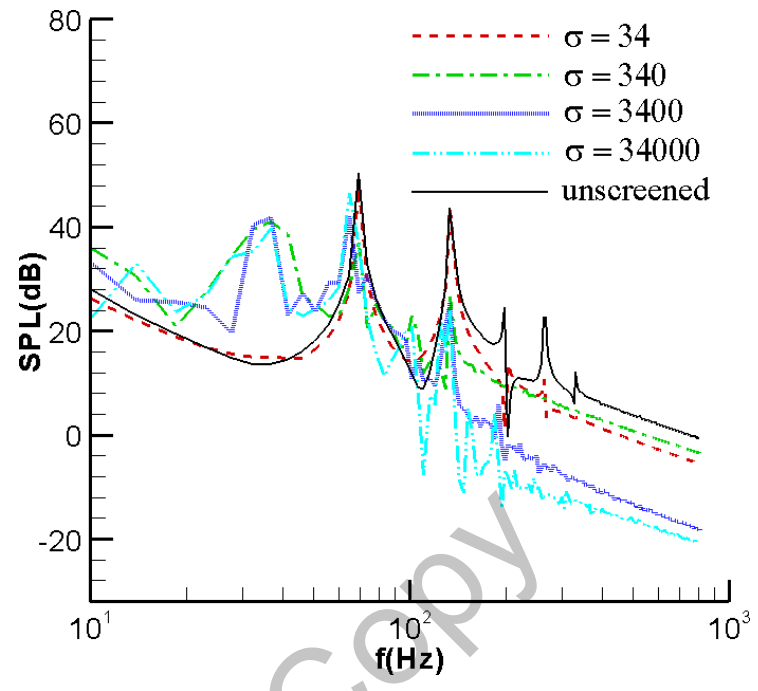

(b) $0.5 \mathrm{D}$

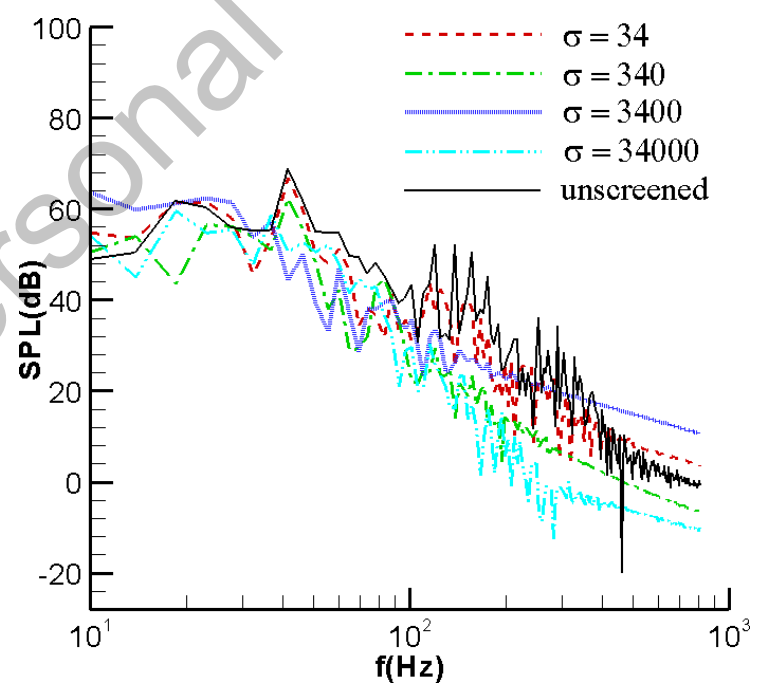

(d) $2.0 \mathrm{D}$

Fig. 7. SPL at the center of the windscreen (a) $0.2 \mathrm{D}$ (b) $0.5 \mathrm{D}$ (c) 1.0D (d) $2.0 \mathrm{D}$ (all values are dimensional).

For Case (c) with upstream cylinder diameter 1.0D, the second lowest flow resistivity windscreen $\left(\sigma=340 \mathrm{~Pa} \cdot \mathrm{s} / \mathrm{m}^{2}\right)$ performs better around $40 \mathrm{~Hz}$, and the highest flow resistivity windscreen $\left(\sigma=34000 \mathrm{~Pa} \cdot \mathrm{s} / \mathrm{m}^{2}\right)$ performs better at 100-200 Hz. For Case (d) with the upstream cylinder diameter $2.0 \mathrm{D}$, the windscreen with $\sigma=340 \mathrm{~Pa} \cdot \mathrm{s} / \mathrm{m}^{2}$ is better around $20 \mathrm{~Hz}$, the one with $\sigma=3400 \mathrm{~Pa} \cdot \mathrm{s} / \mathrm{m}^{2}$ does in the $50-60 \mathrm{~Hz}$ range, and the highest flow resistivity windscreen $\left(\sigma=34000 \mathrm{~Pa} \cdot \mathrm{s} / \mathrm{m}^{2}\right)$ performs better in the $100-300 \mathrm{~Hz}$ range.

Given that the real wind turbulence is a high Reynolds number flow composed of vortical structures spanning a large range of sizes, combining the four different upstream cylinder 


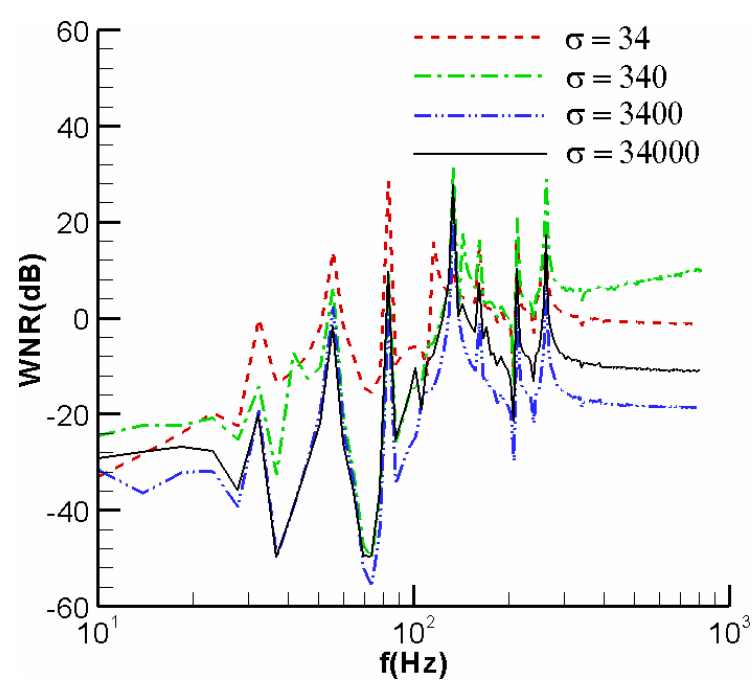

(a) $0.2 \mathrm{D}$

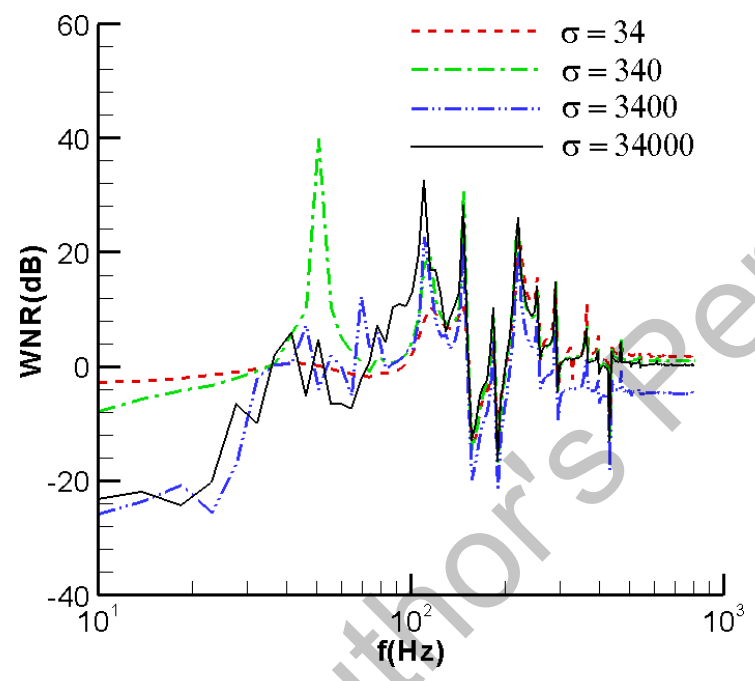

(c) $1.0 \mathrm{D}$

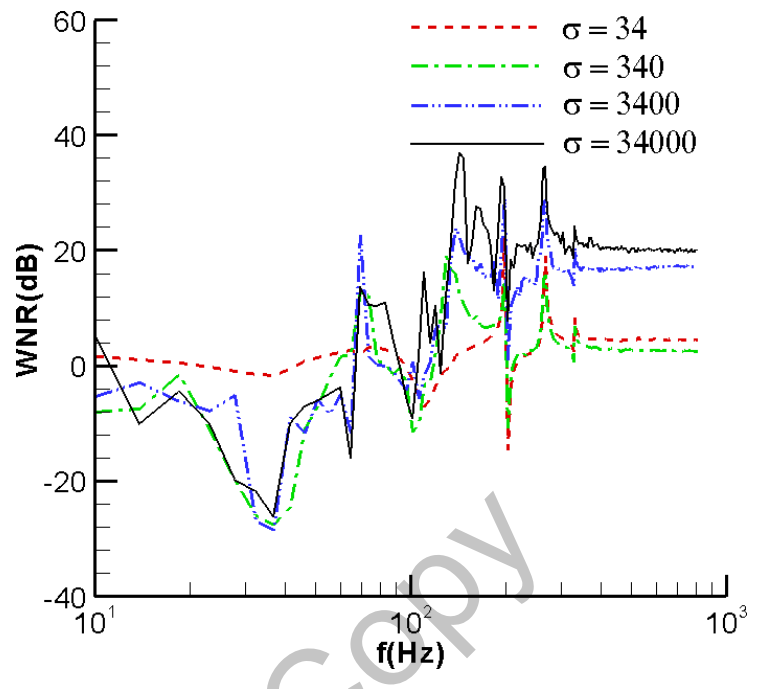

(b) $0.5 \mathrm{D}$

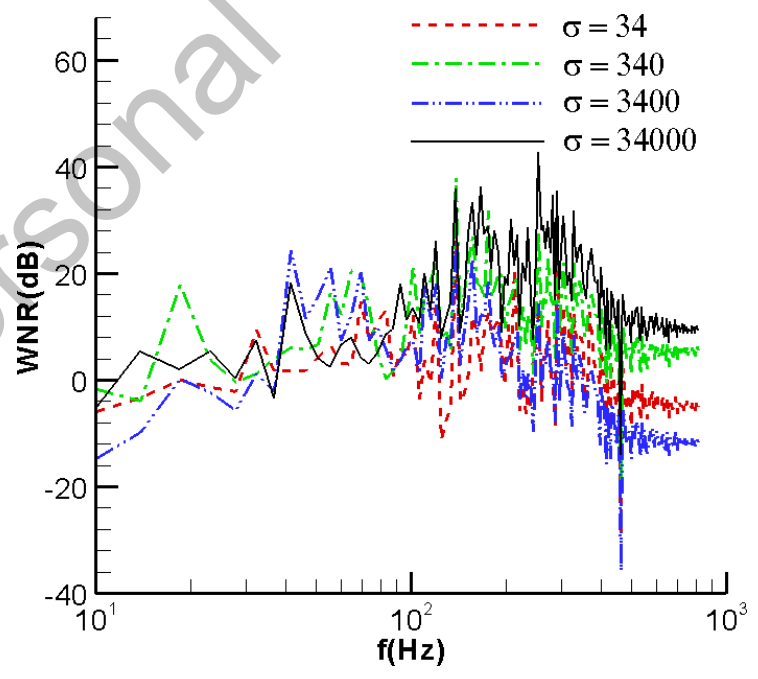

(d) $2.0 \mathrm{D}$

Fig. 8. Wind noise reduction (WNR) between unscreened microphone center and screened microphone center (a) $0.2 \mathrm{D}$ (b) $0.5 \mathrm{D}$ (c) $1.0 \mathrm{D}$ (d) $2.0 \mathrm{D}$ (all values are dimensional).

cases together yields the average WNR level for different levels of turbulence for each windscreen flow resistivity. The average WNR is computed as the arithmetic average of the WNR values from the four cases in Fig. 8. Figure 9 shows the average wind noise reduction between the unscreened microphone and the screened microphone. For the low flow resistivity $\left(\sigma=34 \mathrm{~Pa} \cdot \mathrm{s} / \mathrm{m}^{2}\right)$, the highest WNR levels are about $7 \mathrm{~dB}$ at $80 \mathrm{~Hz}$, and $6 \mathrm{~dB}$ at $300 \mathrm{~Hz}$. For the second lowest flow resistivity $\left(\sigma=340 \mathrm{~Pa} \cdot \mathrm{s} / \mathrm{m}^{2}\right)$, the highest WNR levels are about $6 \mathrm{~dB}$ at $50 \mathrm{~Hz}$, about $14 \mathrm{~dB}$ at $120 \mathrm{~Hz}$, and about $12 \mathrm{~dB}$ at $300 \mathrm{~Hz}$. For the medium flow resistivity $\left(\sigma=3400 \mathrm{~Pa} \cdot \mathrm{s} / \mathrm{m}^{2}\right)$, the high WNR levels are about $10 \mathrm{~dB}$ at $120 \mathrm{~Hz}$, and 


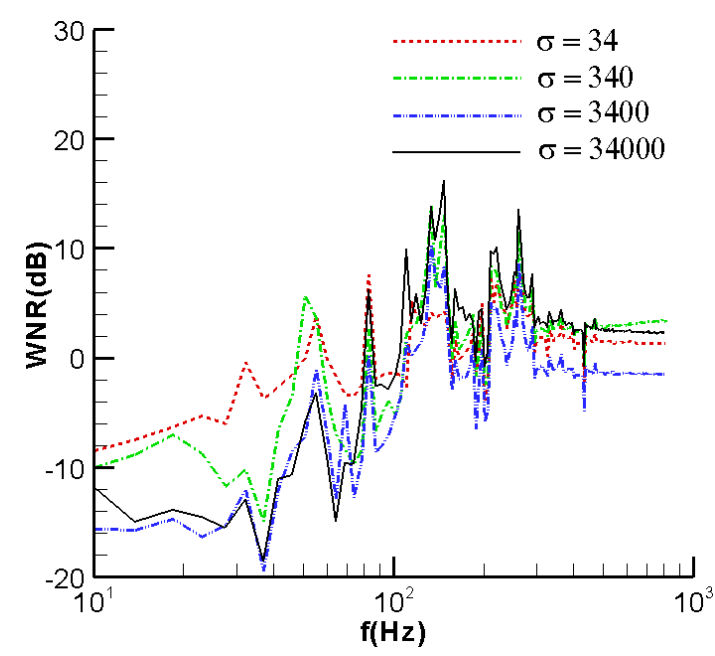

Fig. 9. Average wind noise reduction (WNR) between the unscreened microphone and the screened microphone. (All values are dimensional.)

$8 \mathrm{~dB}$ at $300 \mathrm{~Hz}$. For the highest flow resistivity $\left(\sigma=34000 \mathrm{~Pa} \cdot \mathrm{s} / \mathrm{m}^{2}\right)$, the highest WNR levels are about $16 \mathrm{~dB}$ at $120 \mathrm{~Hz}$, and $14 \mathrm{~dB}$ at $300 \mathrm{~Hz}$. This means that, in the low frequency range (less than $100 \mathrm{~Hz}$ ), windscreens of low flow resistivity are effective in reducing noise, while those with high flow resistivity are not. At the high frequency range (greater than $100 \mathrm{~Hz}$ ), windscreens with high flow resistivity provide more reduction than those with low flow resistivity.

It should be noted that the study in this paper is for a cylindrical windscreen with a long axis so that the two-dimensional approximation can be made. For realistic three-dimensional turbulent flow and three-dimensional windscreens, the frequency range can be shifted due to three-dimensional effects. However, we tested some cases of flow over spheres and found out that the physical mechanisms revealed in this study and the relative noise reduction trend did not change significantly.

\section{Conclusion}

This paper examined the wind noise reduction effect of porous-medium windscreens. A highorder simulation scheme was employed to improve the accuracy at the interface between the open air and porous medium. We used a coupled computation for flow both outside and inside the windscreen. Windscreens with varying flow resistivities were tested with different incoming wind turbulence conditions as induced by different sizes of upstream cylinders. For low frequency turbulence, low flow resistivity windscreen materials produce more noise reduction than high flow resistivity materials. As a matter of fact, for very low frequency turbulence (below $20 \mathrm{~Hz}$ ), windscreens actually enhance the wind noise, although the impact is less adverse for low flow resistivity windscreen materials. For high frequency turbulence, the opposite behavior was observed: high flow resistivity materials produce more 
noise reduction than low flow resistivity materials. The mechanism of wind-noise reduction is the ability of windscreens to diffuse the vortical structures in the turbulent flow.

\section{Acknowledgments}

This research was partially supported by the US Army Engineer Research and Development Center under contract W913E7-07-C-0004 when the first two authors were at Kansas State University.

\section{References}

1. V. E. Ostashev, D. K. Wilson, L. Liu, D. F. Aldridge, N. P. Symons and D. H. Marlin, Equations for finite-difference, time-domain simulation of sound propagation in moving inhomogeneous media and numerical implementation, J. Acoust. Soc. Am. 117 (2005) 503.

2. D. K. Wilson, S. L. Collier, V. E. Ostashev, D. F. Aldridge, N. P. Symons and D. H. Marlin, Time-domain modeling of the acoustic impedance of porous surfaces, Acta Acustica United With Acustica 92 (2006) 965.

3. C. Zwikker and C. W. Kosten, Sound Absorbing Materials (Elsevier, New York, 1949), pp. 18-24.

4. S. Bhattacharyya, S. Dhinakaran and A. Khalili, Fluid motion around and through a porous cylinder, Chem. Eng. Sc. 61 (2006) 4451.

5. A. Harten, B. Engquist, S. Osher and S. Chakravarthy, Uniformly high-order accurate nonoscillatory schemes, J. Comput. Phys. 71 (1987) 231.

6. C. W. Shu and S. Osher, Efficient implementation of nonoscilatory shock capturing schemes, J. Comput. Phys. 83 (1988) 32.

7. T. Kawamura, H. Takami and K. Kuwahara, New higher-order upwind scheme for incompressible Navier-Stokes equations, Lecture Notes in Phys. 218 (1985) 291.

8. Y. Cho, J. Chopra and P. J. Morris, Immersed boundary method for compressible high-Reynolds number viscous flow around moving bodies, 45th AIAA Aerospace Sciences Meeting, AIAA2007125 (Reno, NV, 2007).

9. N. Zhang and Z. C. Zheng, An improved direct-forcing immersed-boundary method for finite difference applications, J. Comput. Phys. 221 (2007) 250.

10. R. H. Kraichnan, Pressure fluctuations in turbulent flow over a flat plate, J. Acoust. Soc. Am. 28 (1956) 378.

11. D. K. Wilson, V. E. Ostashev, S. L. Collier, N. P. Symons, D. F. Aldridge and D. H. Marlin, Time-domain calculations of sound interactions with outdoor ground surfaces, Appl. Acoust. 68 (2007) 173.

12. S. V. Patankar, Numerical Heat Transfer and Fluid Flow (Taylor \& Francis, 1980), pp. 83-85.

13. P. N. Swarztrauber and R. A. Sweet, Algorithm 541, efficient FORTRAN subprograms for the solution of separable elliptic partial differential equations, ACM Trans. on Math. Software 5 (1979) 352.

14. Y. H. Tseng and J. H. Ferziger, A ghost-cell immersed boundary method for flow in complex geometry, J. Comput. Phys. 192 (2003) 593.

15. A. S. Grove, F. H. Shair, E. E. Peterson and A. Acrivos, An experimental investigation of the steady separated flow past a circular cylinder, J. Fluid Mech. 19 (1964) 60.

16. G. H. Goedecke, V. E. Ostashev, D. K. Wilson and H. J. Auvermann, Quasi-wavelet model of von karmon spectrum of turbulent velocity fluctuations, Boundary-Layer Meteorology 112 (2004) 33 .

17. D. K. Wilson, V. E. Ostashev, G. H. Goedecke and H. J. Auvermann, Quasi-wavelet calculations of sound scattering behind barriers, Appl. Acoust. 65 (2004) 605. 